\title{
Erratum: PTEN deletion drives acute myeloid leukemia resistance to MEK inhibitors
}

\author{
Amanda M. Smith ${ }^{1,4, *}$, Christine R.C. Zhang ${ }^{1,4, *}$, Alexandre S. Cristino ${ }^{1,6}$, John P. \\ Grady $^{1,5}$, J. Lynn Fink ${ }^{1}$ and Andrew S. Moore ${ }^{1,2,3}$ \\ ${ }^{1}$ The University of Queensland Diamantina Institute, The University of Queensland, Woolloongabba, Australia \\ ${ }^{2}$ Oncology Services Group, Queensland Children's Hospital, South Brisbane, Australia \\ ${ }^{3}$ Child Health Research Centre, The University of Queensland, South Brisbane, Australia \\ ${ }^{4}$ Current address: Washington University in Saint Louis, Saint Louis, Missouri, United States of America \\ ${ }^{5}$ Current address: Garvan Institute of Medical Research, Darlinghurst, Australia \\ ${ }^{6}$ Current address: Griffith Institute for Drug Discovery, Brisbane Innovation Park, Nathan, Australia \\ *These authors contributed equally to this work
}

Published: January 21, 2020

Copyright: Smith et al. This is an open-access article distributed under the terms of the Creative Commons Attribution License 3.0 (CC BY 3.0), which permits unrestricted use, distribution, and reproduction in any medium, provided the original author and source are credited.

This article has been corrected: During production, the 4th affiliation was mistakenly added to the name of the last author, Dr. Andrew S. Moore. The proper affiliation information is shown below.

Original article: Oncotarget. 2019; 10:5755-5767. https://doi.org/10.18632/oncotarget.27206

\footnotetext{
Amanda M. Smith ${ }^{1,4, *}$, Christine R.C. Zhang ${ }^{1,4, *}$, Alexandre S. Cristino ${ }^{1,6}$, John P. Grady ${ }^{1,5}$, J. Lynn Fink ${ }^{1}$ and Andrew S. Moore ${ }^{1,2,3}$

${ }^{1}$ The University of Queensland Diamantina Institute, The University of Queensland, Woolloongabba, Australia

${ }^{2}$ Oncology Services Group, Queensland Children's Hospital, South Brisbane, Australia

${ }^{3}$ Child Health Research Centre, The University of Queensland, South Brisbane, Australia

${ }^{4}$ Current address: Washington University in Saint Louis, Saint Louis, Missouri, United States of America

${ }^{5}$ Current address: Garvan Institute of Medical Research, Darlinghurst, Australia

${ }^{6}$ Current address: Griffith Institute for Drug Discovery, Brisbane Innovation Park, Nathan, Australia

*These authors contributed equally to this work
} 